\title{
The Need for Further Fine-Grained Distinctions in Discussions of Authenticity and Deep Brain Stimulation
}

Gilbert et al. present important new qualitative data regarding patients' experiences of self-estrangement following Deep Brain Stimulation (DBS) in the treatment of Parkinson's Disease (PD). ${ }^{1}$ Philosophical discussions of selfestrangement (and the related concept of authenticity) are commonplace in the neuroethical literature concerning $\mathrm{DBS},{ }^{2}$ but empirical data concerning the phenomenon are essential to ensuring that philosophical discussions of estrangement and authenticity are grounded in clinical reality. Moreover, such data highlight important distinctions that add nuance to philosophical discussions. For instance, Gilbert et al. draw an illuminating distinction between deteriorative estrangement and restorative estrangement. Acknowledging that there can be such qualitatively different kinds of self-estrangement raises an important challenge for neuroethical arguments that implicitly assume that self-estrangement is a source of disvalue.

We shall highlight three further distinctions pertaining to authenticity and selfestrangement that we believe should be adopted in future research, to aid our understanding of these complex phenomena. The first distinguishes between feelings of estrangement arising from an inability to act in accordance with one's authentic motivational sates, and feelings of estrangement from one's motivational states themselves. As we shall explain, the significance of this distinction is evidenced by Gilbert et al.'s data.

Consider the following statement from patient 16:

[Parkinson's Disease] really takes over. [...] I couldn't work which was a

big part of my identity. [...] If I didn't have the device I'd probably be dead right now... I think that [DBS] does change you as a person. ${ }^{3}$

As Gilbert et al. point out, their data suggest a strong correlation between postoperative estrangement and how patients preoperatively perceive themselves with respect to their illness; if, like patient 16 , one feels alienated from one's illness, then one is likely to experience post-operative feelings of estrangement. However, Gilbert et al. do not explain whether there is a correlation between kinds of preoperative perceptions of illness, and particular qualitative types of estrangement.

Notice that patient 16 implies that PD threatens their sense of authenticity partly because it renders them unable perform certain actions that were incorporated into their self-understanding. It seems that this kind of preoperative perception of PD will most plausibly give rise to restorative rather than deteriorative postoperative estrangement, insofar as DBS will serve to restore the patient's ability to perform the actions in question.

In contrast, DBS is commonly assumed to be associated with deteriorative self-estrangement because recipients sometimes develop different emotional responses or motivational states following stimulation. ${ }^{4}$ Indeed, patient 04 experiences deteriorative estrangement because his/her children no longer recognize

\footnotetext{
${ }^{1}$ Gilbert et al., 'I Miss Being Me: Phenomenological Effects of Deep Brain Stimulation.'

${ }^{2}$ Kraemer, 'Me, Myself and My Brain Implant'; Nyholm and O'Neill, 'Deep Brain Stimulation, Continuity over Time, and the True Self'.

${ }^{3}$ Gilbert et al., 'I Miss Being Me: Phenomenological Effects of Deep Brain Stimulation.'

${ }^{4}$ Kraemer, 'Me, Myself and My Brain Implant'.
} 
him/her due to his/her increased impulsivity. Similarly, in the psychiatric context, individuals may feel alienated from their preoperative disease state because it affects their emotional responses and motivational states, rather than their physical abilities.

Accordingly, we can distinguish between, (A) feelings of estrangement that arise from an inability to act in accordance with one's authentic motivational states, and (B) feelings of estrangement from new emotional and motivational states that compete with one's pre-existing authentic states. Why does this distinction matter? First, it may plausibly help to elucidate the ambivalent feelings of estrangement of some patients. Consider, for example, patient 13. This patient states that "[DBS] has allowed me to return almost to the person I was before", but also notes that stimulation leads to intermittent uncontrollable emotional sensitivity, which leads them to feel like "I had lost my true self". Acknowledging the above distinction might help to allow us to accommodate this sort of ambivalence. Second, it might be argued that these different dimensions of authenticity bear different evaluative weight. If, for instance, an individual's emotional and motivational states are more foundational to their authentic self than their physical ability to act effectively in accordance with their authentic motivational states, then the former should arguably be prioritised over the latter.

It might be thought that the above distinction is merely a restatement of Gilbert et al.'s distinction between restorative and deteriorative estrangement. However, whilst there is a degree of overlap in the case of PD, this is partly a function of the fact that the aim of DBS in PD is to alleviate motor symptoms. In this context, the effects of DBS on the patient's motivational and emotional states are either indirect (in the way that Gilbert et al highlight), or off-target side effects. In contrast, psychiatric uses of DBS might be intended to directly alter the recipient's motivational and emotional states. ${ }^{5}$ Depending on the patient's pre-operative experience of their illness, such changes might plausibly lead to feelings of either restorative or deteriorative estrangement in Gilbert et al.'s terms. As such, the two distinctions are not co-extensive.

We now highlight two further distinctions that we believe add further nuance to discussions pertaining to the phenomena of estrangement and authenticity. These distinctions might usefully be investigated in further empirical research.

In a forthcoming paper, we draw a distinction between the authenticity of an individual's traits, and the authenticity of their values. ${ }^{6}$ We understand a value to refer to an agent's endorsement of some end as good in a reason-implying sense. If one values $x$, then one believes that one has a reason to pursue $x$; values are thus inherently normative. In contrast, we understand an individual's traits in a nonnormative sense; they are motivational and emotional characteristics that the individual is prone to manifest. Crucially, whilst individuals may commonly exhibit traits that reveal their values, an individual's values do not determine their traits; indeed, we often fail to live up to our values in our behaviours and emotional responses.

This distinction can be usefully invoked when considering deteriorative estrangement following DBS. Some cases of deteriorative self-estrangement may result from a discrepancy between the individual's values and their traits following DBS. For instance Doshi and Bhargava report a case of an individual developing

\footnotetext{
${ }^{5}$ Maslen, Pugh, and Savulescu, 'The Ethics of Deep Brain Stimulation for the Treatment of Anorexia Nervosa'.

${ }^{6}$ Pugh, Maslen, and Savulescu, ‘Deep Brain Stimulation, Authenticity and Value'.
} 
hyper-sexuality following DBS for PD. These hypersexual urges were incongruous with the patient's own values. ${ }^{7}$ Yet, deteriorative estrangement following DBS may have a deeper source if stimulation affects the patient's values themselves. For instance, Voon et al have observed that increased apathy can be an unintended postoperative symptom following DBS of the subthalamic nucleus; ${ }^{8}$ such increases in apathy might plausibly have a significant effect on the patient's values. We argue that such effects pose a deeper and more fundamental challenge to authenticity than changes to individual traits. ${ }^{9}$

However, Gilbert et al. might challenge the utility of this distinction, in light of their particular conception of estrangement. Notably, Gilbert et al. frame estrangement in non-cognitive phenomenological terms; self-estrangement on this framing is a kind of subjective feeling or emotional experience with a certain qualitative character. On such an approach, the above distinction may be thought to lack practical value, because patients who undergo a change in values following DBS may not postoperatively experience feelings of self-estrangement. By the lights of their new values, they may feel they are living authentically despite undergoing a radical change in their values.

Here though, it is important to draw a third distinction by contrasting Gilbert et al.'s phenomenological approach with approaches to authenticity in the philosophical literature that include cognitive, and even non-subjective elements. On such accounts, an individual's feeling of estrangement or authenticity may not exhaust the relevant considerations in this context. For instance, on Marya Schetmann's prominent conception of narrative identity, a self-narrative must admit of rational articulation and correspond to observable facts about reality. ${ }^{10}$ On this approach, an individual can be alienated from her real self and the values that partly constitute it, even if she does not phenomenologically experience estrangement. Such an approach suggests that parties other than the individual might be appropriately included in assessments of authenticity.

Rather than understanding the distinction between non-cognitive and cognitive conceptions of authenticity as identifying competing views, we believe that a rich understanding of authenticity and estrangement should incorporate both of these elements. However, when the two are in conflict, our decisions about which element to prioritise will depend to a large extent on how our understanding of the nature of authenticity's contribution to wellbeing, and its role in personal autonomy. Future research into patients' understanding and adoptions of these different conceptions would bring further clarity in this area.

\footnotetext{
${ }^{7}$ Doshi and Bhargava, 'Hypersexuality Following Subthalamic Nucleus Stimulation for Parkinson's Disease'.

${ }^{8}$ Voon et al., 'Deep Brain Stimulation'.

${ }^{9}$ Pugh, Maslen, and Savulescu, 'Deep Brain Stimulation, Authenticity and Value'.

${ }^{10}$ Schechtman, The Constitution of Selves. See Baylis, "“I Am Who I Am"' for discussion in the context of DBS. We defend a cognitive approach to authenticity in Pugh, Maslen, and Savulescu, 'Deep Brain Stimulation, Authenticity and Value'.
} 


\section{References}

Baylis, Françoise. " I Am Who I Am": On the Perceived Threats to Personal Identity from Deep Brain Stimulation'. Neuroethics 6, no. 3 (2013): 513-26. doi:10.1007/s12152-011-9137-1.

Doshi, Paresh, and Pranshu Bhargava. 'Hypersexuality Following Subthalamic Nucleus Stimulation for Parkinson's Disease'. Neurology India 56, no. 4 (December 2008): 474-76.

Gilbert, Frederic, E Goddard, JNM Viana, A Carter, and M Horne. 'I Miss Being Me: Phenomenological Effects of Deep Brain Stimulation.' American Journal of Bioethics Neuroscience, forthcoming.

Hope, Tony, Jacinta Tan, Anne Stewart, and John McMillan. 'Agency, Ambivalence and Authenticity: The Many Ways in Which Anorexia Nervosa Can Affect Autonomy'. International Journal of Law in Context 9, no. Special Issue 01 (March 2013): 20-36. doi:10.1017/S1744552312000456.

Kraemer, Felicitas. 'Me, Myself and My Brain Implant: Deep Brain Stimulation Raises Questions of Personal Authenticity and Alienation'. Neuroethics 6, no. 3 (2013): 483-97. doi:10.1007/s12152-011-9115-7.

Maslen, Hannah, Jonathan Pugh, and Julian Savulescu. 'The Ethics of Deep Brain Stimulation for the Treatment of Anorexia Nervosa'. Neuroethics 8, no. 3 (2015): 215-230.

Nyholm, Sven, and Elizabeth O'Neill. 'Deep Brain Stimulation, Continuity over Time, and the True Self'. Cambridge Quarterly of Healthcare Ethics: CQ: The International Journal of Healthcare Ethics Committees 25, no. 4 (October 2016): 647-58. doi:10.1017/S0963180116000372.

Pugh, Jonathan, Hannah Maslen, and Julian Savulescu. 'Deep Brain Stimulation, Authenticity and Value'. Cambridge Quarterly of Healthcare Ethics, Forthcoming.

Schechtman, Marya. The Constitution of Selves. Ithaca ; London: Cornell University Press, 1996.

Voon, Valerie, Cynthia Kubu, Paul Krack, Jean-Luc Houeto, and Alexander I. Tröster. 'Deep Brain Stimulation: Neuropsychological and Neuropsychiatric Issues'. Movement Disorders: Official Journal of the Movement Disorder Society 21 Suppl 14 (June 2006): S305-327. doi:10.1002/mds.20963. 